\title{
First experimental verification of summertime mesospheric momentum balance based on radar wind measurements at $69^{\circ} \mathrm{N}$
}

\author{
M. Placke ${ }^{1}$, P. Hoffmann ${ }^{1}$, and M. Rapp ${ }^{2,3}$ \\ ${ }^{1}$ Leibniz Institute of Atmospheric Physics at the Rostock University, Kühlungsborn, Germany \\ ${ }^{2}$ Deutsches Zentrum für Luft- und Raumfahrt, Institut für Physik der Atmosphäre, Oberpfaffenhofen, Germany \\ ${ }^{3}$ Meteorologisches Institut München, Ludwig-Maximilians-Universität München, Munich, Germany
}

Correspondence to: M. Placke (placke@iap-kborn.de)

Received: 16 July 2015 - Accepted: 24 August 2015 - Published: 10 September 2015

\begin{abstract}
Gravity waves (GWs) greatly influence the background state of the middle atmosphere by imposing their momentum on the mean flow upon breaking and by thus driving, e.g., the upper mesospheric summer zonal wind reversal. In this situation momentum is conserved by a balance between the vertical divergence of GW momentum flux (the so-called GW drag) and the Coriolis acceleration of the mean meridional wind. In this study, we present first quantitative mean annual cycles of these two balancing quantities from the medium frequency Doppler radar at the polar site Saura (SMF radar, $69^{\circ} \mathrm{N}, 16^{\circ} \mathrm{E}$ ). Three-year means for 2009 through 2011 clearly show that the observed zonal momentum balance between 70 and $100 \mathrm{~km}$ with contributions from GWs only is fulfilled during summer when GW activity is strongest and more stable than in winter. During winter, the balance between GW drag and Coriolis acceleration of the mean meridional wind is not existent, which is likely due to the additional contribution from planetary waves, which are not considered by the present investigation. The differences in the momentum balance between summer and winter conditions are additionally clarified by 3 -month mean vertical profiles for summer 2010 and winter 2010/2011.
\end{abstract}

Keywords. Ionosphere (wave propagation) - meteorology and atmospheric dynamics (middle atmosphere dynamics; waves and tides)

\section{Introduction}

Atmospheric gravity waves (hereafter: GWs) propagate vertically and horizontally from their tropospheric source regions to the mesosphere and lower thermosphere (MLT,
$80-100 \mathrm{~km}$ ), which is a main region of GW breaking and hence associated with the deposition of GW momentum and energy. Thus, GWs are responsible for the vertical coupling between different atmospheric layers. Vertical propagation of GWs is in principle only possible if the waves move against the mean flow and do not reach any critical levels where they are filtered by the background wind. Dynamical processes like momentum deposition by breaking GWs lead to changes of the temperature and wind field. Based on the quasi-geostrophic transformed Eulerian-mean (TEM) equations on a beta-plane (see, e.g., Andrews et al., 1987), the momentum balance in zonal direction is given as

$$
\frac{\partial u}{\partial t}-f \cdot v=\frac{1}{\rho_{0}} \nabla \cdot \boldsymbol{F}+X \equiv D_{u} .
$$

Here, $u$ and $v$ are the zonally averaged zonal wind velocity and residual mean meridional circulation, $t$ is time, and $f=2 \Omega \sin \phi$ is the Coriolis frequency, which includes the rotation rate of the Earth $\Omega$ and the latitude $\phi$. Note that for simplicity, mean values are denoted without overbars in the text. The Eliassen-Palm flux divergence $\boldsymbol{\nabla} \cdot \boldsymbol{F}$ per temporal mean density $\rho_{0}$ represents together with $X$ the zonal GW drag $D_{u}$, which is the GW momentum deposition into the zonal wind field, i.e., a zonal force per unit mass on the zonal-mean flow. The Eliassen-Palm flux $\boldsymbol{F}$ contains contributions from both planetary waves as well as small-scale GWs, and $X$ represents all further contributions to the mean zonal force per unit mass associated with GWs and other small-scale disturbances. For a steady-state atmosphere with vanishing time derivatives, which is the case, e.g., during the solstices, the zonal momentum balance in the extratropical MLT is primarily given between the zonal mean GW forcing and the zonal 
mean Coriolis force as for instance described by Liu et al. (2009). For summer conditions planetary waves play a minor role in the mesosphere because tropospheric excited planetary waves cannot propagate up to mesospheric heights due to the Charney-Drazin criterion (Charney and Drazin, 1961). Hence, $\boldsymbol{F}$ is primarily determined by the contribution from GWs. Consequently, the summer momentum balance in zonal direction is between the mean flow acceleration due to the divergence of the GW momentum flux and the negative Coriolis acceleration of the mean meridional wind:

$-f \cdot v \approx D_{u}=-\frac{1}{\rho_{0}} \cdot \frac{\partial\left(\rho u^{\prime} w^{\prime}\right)}{\partial z}$.

Here, $u^{\prime} w^{\prime}$ and $\rho$ are the temporal mean vertical flux of zonal momentum and the temporal mean density at $1 \mathrm{~km}$ above and below a reference height with a temporal mean density $\rho_{0}$. During wintertime, however, planetary waves can propagate into the mesosphere and contribute to $\boldsymbol{F}$ such that the relation given in Eq. (2) is not fulfilled (see Andrews et al., 1987, for more details). Based on Eq. (2) it is obvious that the vertical divergence of the vertical flux of zonal momentum that is imposed by breaking GWs on the mean background flow drives the residual meridional summer-to-winter-pole circulation. Subsequently, for reasons of mass conservation, this meridional flow needs to be balanced, which leads to a residual upwelling and hence adiabatic cooling over the summer pole as well as a residual downwelling and adiabatic warming over the winter pole. This results in very low temperatures in the summer mesopause and the existence of the warm winter stratopause (e.g., Holton and Alexander, 2000). This chain of causality has first been introduced by, e.g., Lindzen (1981) and is described in detail by, e.g., Dunkerton and Butchart (1984), Andrews et al. (1987), and Becker (2011). First and most realizations of this mechanism are based on parametrization in models (Lindzen, 1981; Holton, 1982). For reviews we refer the reader to Holton and Alexander (2000), who give a general description, to Alexander et al. (2010), who give an overview on the current status of GW effects in atmospheric models and observations, and to a recently published first comparison between GW absolute momentum fluxes from climate models, high-resolution models, and fluxes derived from global satellite observations (Geller et al., 2013).

Based on the technique by Vincent and Reid (1983), first direct observations of the upper mesospheric momentum balance were performed, e.g., by Reid and Vincent (1987) with the Buckland Park MF radar near Adelaide. However, they only focussed on some selected case studies for few days throughout the year. Another case study of GW flux measurements using MF radar interferometry was presented by Hall et al. (1992) at Saskatoon. These authors could not find a balance between zonal GW deceleration and Coriolis acceleration, probably owing to their limited time series of only 17 days. Later, Sato (1994) studied the stratospheric vertical momentum flux, variance and drag of the background wind with the MU radar and found evidence for a strong interaction between inertioGWs and the background wind field in summer and winter. A further attempt to evaluate the momentum balance in the mesosphere has been presented by Frame et al. (2000). They used horizontal winds from two MF radars near Adelaide, Australia, and Christchurch, New Zealand, in connection with satellite temperatures in order to resolve the momentum equations appropriate for large-scale fluid flow on a sphere. Caused by the limitations of data availability and vertical extent, and using additional theoretical assumptions they estimated and discussed the mean momentum balance for only 1 month at an altitude of $80 \mathrm{~km}$. Momentum balance results of Liu et al. (2009) were based on two different simplifications derived from the full momentum equation in zonal direction using WACCM3 model results for December conditions. Whereas the results of both methods were comparable in the Southern Hemisphere (summer), differences occurred mainly in the Northern Hemisphere (winter). Based on the model findings, ground-based lidar wind measurements as well as winds calculated from 61 days of TIDI measurements were used for an indirect estimation of the GW forcing. In contrast to the extensive method presented by Frame et al. (2000) resolving the complete momentum equations and to the indirect method proposed by Liu et al. (2009) to estimate the GW forcing, we use in our present study a direct way to estimate the momentum balance from measurements with the Saura MF Doppler radar (hereafter: SMF radar) at polar latitudes (Singer et al., 2008). This article is organized as follows: in Sect. 2 the SMF radar and the used experimental data are introduced briefly. The annual variation of the mesospheric momentum balance as given in Eq. (2) is then checked and discussed in Sect. 3 on the basis of 3 years of observations, i.e., from 2009 to 2011. With seasonal mean vertical profiles for summer and winter the balancing quantities are regarded quantitatively including their SDs (standard deviations). Conclusions are given in Sect. 4.

\section{Observational data}

The database for the present study consists of observations with the unique, narrow beam SMF radar that is located on the northern Norwegian island of Andøya $\left(69^{\circ} \mathrm{N}, 16^{\circ} \mathrm{E}\right)$. The benefit of this radar is continuous high-quality measurements over a broad height range with high temporal and vertical resolution during all seasons. The SMF radar transmits and receives electromagnetic signals with a Mills Cross antenna of 29 crossed dipoles at a frequency of $3.17 \mathrm{MHz}$ (see Singer et al., 2008, for details). Two coplanar tilted radar beams are transmitted successively in different spatial directions with a fixed off-zenith angle of $6.8^{\circ}$. For any pair of opposite radar beams, the radial wind velocity is measured within $2 \mathrm{~min}$. That means the measurement for four spatial directions (two 

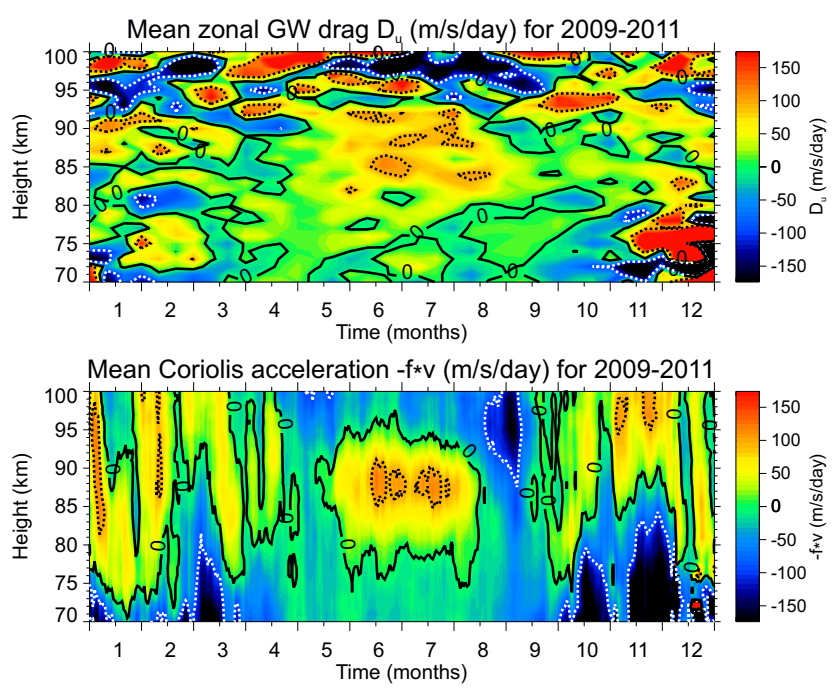

Figure 1. Height-time cross sections of the 3-year mean zonal GW drag $D_{u}$ and negative Coriolis acceleration $-f \cdot v$ from the SMF radar for 2009 through 2011. Zero contour lines are indicated by black solid lines. Contours of negative and positive values are shown with intervals of $100 \mathrm{~m} \mathrm{~s}^{-1} \mathrm{day}^{-1}$ with dotted white and black lines, respectively. The GW drag values are averages over 20 days, shifted by 10 days. The Coriolis acceleration is calculated as averages over 7 days, shifted by 1 day.

coplanar beam measurements perpendicular to each other) takes $4 \mathrm{~min}$. This is the temporal resolution for the analysis of the zonal $(u)$ and the meridional wind $(v)$, which are determined from the measured radial wind velocity (see Placke et al., 2015, for details of the wind analysis). This radar configuration has been used since the middle of 2007. Vertically, the SMF radar observations range from about 60 to $100 \mathrm{~km}$ with a vertical resolution of $1 \mathrm{~km}$.

GW momentum fluxes are determined from the radial wind velocity variations of the coplanar radar beams by applying the method by Vincent and Reid (1983). The implementation of this method and the application of data selection criteria for obtaining statistically meaningful momentum flux results has been done according to Placke et al. (2015). For validating the mesospheric momentum balance, the vertical GW momentum flux divergence $D_{u}$ is calculated using density values from the NRLMSISE-00 empirical atmospheric model (Picone et al., 2002), which are available daily with an altitude resolution of $1 \mathrm{~km}$.

\section{Verification of the momentum balance}

\subsection{Mean annual variation}

The summer mesospheric momentum balance as theoretically expected from Eq. (2) is now checked on the basis of the experimental data from the SMF radar. Figure 1 shows the mean annual variation of zonal GW drag $D_{u}$ and negative Coriolis acceleration of the mean meridional wind $-f \cdot v$ between 70 and $100 \mathrm{~km}$ altitude for 3 years (2009 through 2011). Consistent with the calculations of the vertical flux of zonal momentum $u^{\prime} w^{\prime}$ and of the meridional wind $v$ in Placke et al. (2015), the zonal GW drag is shown as running averages over 20 days, which are shifted by 10 days, and the Coriolis acceleration is shown as running averages over 7 days, which are shifted by 1 day. With these averaging intervals the high temporal variability of the wind field is taken into account and the momentum flux is calculated for a sufficiently long time span in order to obtain reliable values (see, e.g., Kudeki and Franke, 1998). It is noticeable that both quantities have an almost homogeneous and comparable structure during summer, but more heterogeneous and differing patterns during winter. As described in Sect. 1, the contribution from both planetary waves and GWs to the mesospheric momentum balance depends on the season. During summer the influence of planetary waves is minor such that the momentum balance as given in Eq. (2) is quantitatively fulfilled, i.e., from end of May until middle of August. $D_{u}$ and $-f \cdot v$ vary predominantly around $0 \mathrm{~m} \mathrm{~s}^{-1}$ day $^{-1}$ below $78 \mathrm{~km}$, have positive values with maxima of about $120 \mathrm{~m} \mathrm{~s}^{-1}$ day $^{-1}$ between 78 and $93 \mathrm{~km}$, and are predominantly negative above. Further, the observed mean characteristics of the MLT dynamics agree reasonably well with the zonal-mean behavior as for instance discussed in Hoffmann et al. (2010). In contrast, the winter season is mainly dominated by the presence of planetary waves, which disturb the propagation of GWs (see, e.g., Pancheva and Mitchell, 2004; Matthias et al., 2013). This additional influence of the planetary waves on the momentum balance is not considered by the SMF radar observations in this study. Hence, the winter zonal GW drag from Fig. 1 no longer balances the negative Coriolis acceleration of the mean meridional wind. Both quantities vary strongly in height and time with positive and negative maxima of similar magnitudes as in summer. Thereby, $D_{u}$ shows no clear pattern whereas $-f \cdot v$ is predominantly positive above $80 \mathrm{~km}$ and negative beneath for end of September through April. During May and from middle of August through middle of September, $-f \cdot v$ is negative over the whole shown altitude range. Note that as described in Placke et al. (2015), extreme values at the lowermost and uppermost heights may be less reliable when compared to values in the central altitude domain (between $\sim 75$ and $95 \mathrm{~km}$ ) because they are calculated from a lower number of radial wind values. Additionally, especially the values between about 70 and $80 \mathrm{~km}$ in winter have higher SDs than in the other heights and seasons.

\subsection{Mean vertical profiles in summer and winter}

In the following, the findings from the height-time cross sections for summer and winter are discussed on the basis of 3-month mean vertical profiles for June through $\mathrm{Au}$ gust 2010 (JJA) and December 2010 through February 2011 

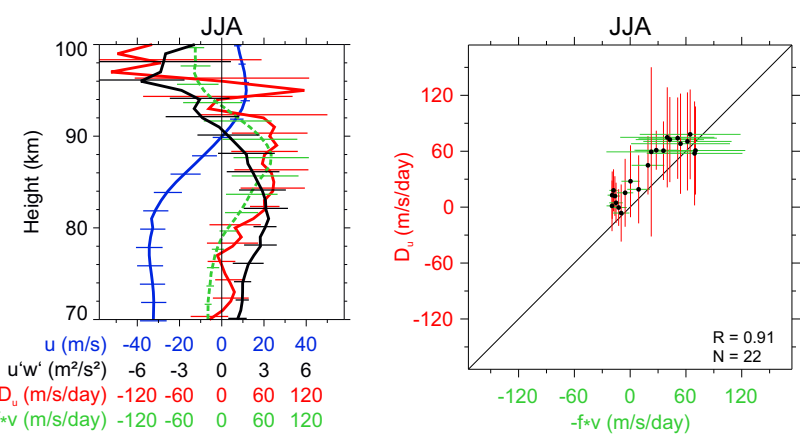

Figure 2. Vertical profiles (left) of 3-month mean zonal wind $u$ (blue), vertical flux of zonal GW momentum $u^{\prime} w^{\prime}$ (black), zonal GW drag $D_{u}$ (red) and negative Coriolis acceleration of the mean meridional wind $-f \cdot v$ (dashed green) from SMF radar measurements in summer 2010. Horizontal lines denote the SD of each quantity at each height and are plotted every $2 \mathrm{~km}$ for simplicity. The scatter plot of $-f \cdot v$ and $D_{u}$ (right) including the SDs for 71 to $92 \mathrm{~km}$ altitude under specification of the correlation coefficient $R$ and the number of values $N$. See text for further information.

(DJF). These results stress the aforementioned magnitudes and errors quantitatively for exemplary periods. The data are calculated on the basis of running averages over 10 days, which are shifted by 1 day for a consistent error estimation of all quantities. The left panels of Figs. 2 and 3 show the mean vertical profiles of zonal wind $u$, vertical flux of zonal GW momentum $u^{\prime} w^{\prime}$, zonal GW drag $D_{u}$, and negative Coriolis acceleration of the mean meridional wind $-f \cdot v$ as well as their corresponding SDs for JJA and DJF. The vertical profiles are complemented by scatter plots (right panels) describing the correlation between $-f \cdot v$ and $D_{u}$. The 3-month mean values of $u$ and $u^{\prime} w^{\prime}$ for summer 2010 in Fig. 2 show the well-known GW-mean flow interaction, which was for instance discussed in detail also for this radar by Placke et al. (2015). Thus, zonal wind and vertical flux of zonal GW momentum are oppositely directed and reverse both in sign around $90 \mathrm{~km}$. That means $u$ is westward directed with magnitudes of approximately $-35 \mathrm{~m} \mathrm{~s}^{-1}$ below $90 \mathrm{~km}$ and it is eastward directed above with maxima around $10 \mathrm{~m} \mathrm{~s}^{-1}$. Simultaneously $u^{\prime} w^{\prime}$ reverses from positive values $\left(\sim 3 \mathrm{~m}^{2} \mathrm{~s}^{-2}\right)$ below $90 \mathrm{~km}$ to negative values (up to $6 \mathrm{~m}^{2} \mathrm{~s}^{-2}$ ) above. The SDs of both quantities vary according to their magnitudes in the particular altitudes. Hence, the SD of $u$ varies between about $\pm 7 \mathrm{~m} \mathrm{~s}^{-1}$ below $90 \mathrm{~km}$ and $\pm 2-3 \mathrm{~m} \mathrm{~s}^{-1}$ above. The corresponding values for $u^{\prime} w^{\prime}$ increase with height from $\pm 1 \mathrm{~m}^{2} \mathrm{~s}^{-2}$ to about $\pm 5 \mathrm{~m}^{2} \mathrm{~s}^{-2}$ in the whole shown altitude range. Overall, these SDs are relatively small owing to the strong and stable GWs in summer. The mean vertical profiles of zonal GW drag $D_{u}$ and negative Coriolis acceleration of the mean meridional wind $-f \cdot v$ for summer 2010 both cover values between $\pm 20 \mathrm{~m} \mathrm{~s}^{-1}$ day $^{-1}$ below $80 \mathrm{~km}$ and maximize in about 60 to $70 \mathrm{~m} \mathrm{~s}^{-1}$ day $^{-1}$ between 82 and $92 \mathrm{~km}$. This is the altitude range where
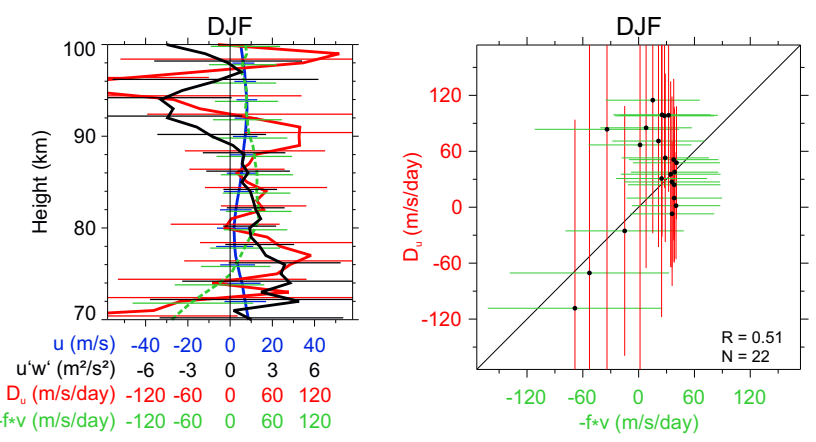

Figure 3. Same as Fig. 2, but for winter 2010/2011.

GW drag maximizes owing to increased GW breaking and hence momentum deposition on the background atmosphere. Above $92 \mathrm{~km}, D_{u}$ varies strongly (between $\pm 120 \mathrm{~m} \mathrm{~s}^{-1} \mathrm{day}^{-1}$ ) and has negative values above $96 \mathrm{~km}$ as observed in the 3-year mean height-time cross sections in Fig. 1. The Coriolis acceleration of the mean meridional wind also reverses to negative values above $93 \mathrm{~km}$, but only in the order of $-40 \mathrm{~m} \mathrm{~s}^{-1}$ day $^{-1}$. Note that overall $D_{u}$ varies more strongly over the whole shown altitude range than $-f \cdot v$ owing to the vertical derivative of $u^{\prime} w^{\prime}$ entering this quantity.

The SD of $-f \cdot v$ is relatively small below $80 \mathrm{~km}$ (about $\pm 8 \mathrm{~m} \mathrm{~s}^{-1} \mathrm{day}^{-1}$ ) when compared to that of $D_{u}$ $\left( \pm 20 \mathrm{~m} \mathrm{~s}^{-1} \mathrm{day}^{-1}\right)$. Between 82 and $90 \mathrm{~km}$, both quantities have comparable SDs of about $\pm 45 \mathrm{~m} \mathrm{~s}^{-1} \mathrm{day}^{-1}$. Above $90 \mathrm{~km}$, the SDs become again smaller for $-f \cdot v$, but much bigger for $D_{u}$ according to the magnitudes of these quantities. These exemplary summer vertical profiles of both quantities from the SMF radar measurements agree quantitatively very well to each other and prove that the summer momentum balance as given in Eq. (2) is fulfilled in the MLT region. Additionally, the corresponding scatter plot of $D_{u}$ versus $-f \cdot v$ for the values from 71 to $92 \mathrm{~km}$ altitude illustrates the very good correlation of both quantities. The diagram covers the same scale range as the vertical profiles. The values show a highly significant correlation with a correlation coefficient of 0.91 . For comparison only, the $1: 1$ line is added. In Fig. 3 the vertical profiles of $u, u^{\prime} w^{\prime}, D_{u}$, and $-f \cdot v$ as well as the scatter plot of $D_{u}$ versus $-f \cdot v$ are shown for winter 2010/2011. As mentioned before, GW propagation is disturbed during the winter season due to the dominance of other kinds of waves like primarily planetary waves. This means that for the momentum balance contributions from planetary waves and GWs need to be taken into account. Consequently, Eq. (2), which covers only the influence of GWs, is not fulfilled. The 3-month mean vertical profiles of zonal wind and vertical flux of zonal momentum do not show an anticorrelation as observed during summer. That is, while $u$ has weak positive values of $\sim 10 \mathrm{~m} \mathrm{~s}^{-1}$ maximum over the whole shown altitude range, $u^{\prime} w^{\prime}$ is positive below $89 \mathrm{~km}$ and predom- 
inantly negative above with magnitudes of $\pm 5 \mathrm{~m}^{2} \mathrm{~s}^{-2}$. The winter zonal GW drag and negative Coriolis acceleration of the mean meridional wind differ more strongly from each other than in summer. The vertical profiles have predominantly negative values below $75 \mathrm{~km}$ and positive values above with exception of $D_{u}$ being again negative between 92 and $97 \mathrm{~km}$. In the central altitude domain, the magnitudes are mainly up to $\sim 100 \mathrm{~m}^{2} \mathrm{~s}^{-2}$ for $D_{u}$ and up to $\sim 40 \mathrm{~m}^{2} \mathrm{~s}^{-2}$ for $-f \cdot v$, respectively. The corresponding scatter plot of $D_{u}$ versus $-f \cdot v$ for 71 to $92 \mathrm{~km}$ clearly demonstrates the strongly scattering values that have a low correlation coefficient of 0.51 only. Overall, all quantities vary more strongly over the whole regarded altitude range in winter than in summer and have higher SDs. Note that the estimated magnitudes are comparable to the findings of Frame et al. (2000), who showed results from May on the Southern Hemisphere that correspond to early winter values in the Northern Hemisphere.

\section{Conclusions and outlook}

Summarizing the findings of the present work, the momentum balance has been verified quantitatively for the first time from local SMF radar observations in the polar summer MLT when GWs play the major role in the mesospheric dynamics. During winter, planetary waves contribute additionally to the momentum balance and can filter GWs. As the planetary wave contribution is not considered by the present investigations using the SMF radar measurements, the momentum balance including GW contributions only is fulfilled in summer but does not exist in winter. These results have clearly been shown from 3-year mean annual cycles of zonal GW drag and Coriolis acceleration of the mean meridional wind. Three-month mean vertical profiles and scatter plots for summer 2010 (JJA) and winter 2010/2011 (DJF) of these quantities, complemented by zonal wind and vertical flux of zonal GW momentum, complete the investigations and take the SDs into account. In summer, a distinct GW-mean flow interaction can be observed with anticorrelated vertical profiles of zonal wind and vertical flux of zonal momentum. At the same time, zonal GW drag and negative Coriolis acceleration of the mean meridional wind have enhanced and comparable magnitudes in the altitude range between 82 and $92 \mathrm{~km}$, where GW breaking and hence momentum deposition on the background atmosphere increase. In contrast, during winter these quantities vary strongly in magnitudes over the altitude range from 70 to $100 \mathrm{~km}$. Zonal wind and vertical flux of zonal momentum reveal no anticorrelation as observed in summer, and the momentum balance requires the information of both GW and planetary wave contributions. The stronger variability during the more disturbed winter conditions also leads to higher SDs of the investigated quantities than in summer.
In future studies, the momentum balance should also be estimated and discussed for similar radar instruments at other latitudes, like the MF radar at the midlatitude site Juliusruh (see, e.g., Keuer et al., 2007). This would allow the definition of the time and height range where the mainly GW-determined momentum balance is fulfilled for different latitudes. Also the latitudinal dependence of the GW drag strength could be proven. Furthermore, the experimental results should be compared qualitatively and quantitatively to model simulations in order to deepen the understanding of the experimentally determined findings.

Acknowledgements. The SMF radar data are available from the authors upon request. This work is part of the project LOCHMES (Long-term changes in the mesosphere) funded by the Leibniz Society (WGL). We thank Erich Becker for collaboration and Gunter Stober for processing and providing the density values of the NRLMSISE-00 empirical atmospheric model.

The topical editor C. Jacobi thanks one anonymous referee for help in evaluating this paper.

\section{References}

Alexander, M. J., Geller, M., McLandress, C., Polavarapu, S., Preusse, P., Sassi, F., Sato, K., Eckermann, S., Ern, M., Hertzog, A., Kawatani, Y., Pulido, M., Shaw, T. A., Sigmond, M., Vincent, R., and Watanabe, S.: Recent developments in gravity-wave effects in climate models and the global distribution of gravitywave momentum flux from observations and models, Q. J. Roy. Meteor. Soc., 136, 1103-1124, doi:10.1002/qj.637, 2010.

Andrews, D. G., Holton, J. R., and Leovy, C. B.: Middle atmosphere dynamics, Academic Press, San Diego, CA, USA, 489 pp., 1987.

Becker, E.: Dynamical Control of the Middle Atmosphere, Space Sci. Rev., 168, 283-314, doi:10.1007/s11214-011-9841-5, 2011.

Charney, J. G. and Drazin, P. G.: Propagation of planetary-scale disturbances from the lower into the upper atmosphere, J. Geophys. Res., 66, 83-109, doi:10.1029/JZ066i001p00083, 1961.

Dunkerton, T. J. and Butchart, N.: Propagation and selective transmission of internal gravity waves in a sudden warming, J. Atmos. Sci., 41, 1443-1460, 1984.

Frame, D. J., Lawrence, B. N., Fraser, G. J., Vincent, R. A., and Dudhia, A.: A new technique for evaluating mesospheric momentum balance utilizing radars and satellite data, Ann. Geophys., 18, 478-484, doi:10.1007/s00585-000-0478-z, 2000.

Geller, M. A., Alexander, M. J., Love, P. T., Bacmeister, J., Ern, M., Hertzog, A., Manzini, E., Preusse, P., Sato, K., Scaife, A. A., and Zhou, T.: A Comparison between Gravity Wave Momentum Fluxes in Observations and Climate Models, J. Climate, 26, 6383-6405, doi:10.1175/JCLI-D-12-00545.1, 2013.

Hall, G. E., Meek, C. E., and Manson, A. H.: MF radar interferometry measurements of fluxes and Coriolis accelerations over Saskatoon $\left(52^{\circ} \mathrm{N}, 107^{\circ} \mathrm{W}\right)$, J. Geophys. Res., 19, 2293-2296, 1992.

Hoffmann, P., Becker, E., Singer, W., and Placke, M.: Seasonal variation of mesospheric waves at northern middle and high latitudes, J. Atmos. Sol.-Terr. Phy., 72, 1068-1079, doi:10.1016/j.jastp.2010.07.002, 2010. 
Holton, J. R.: The Role of Gravity Wave Induced Drag and Diffusion in the Momentum Budget of the Mesosphere, J. Atmos. Sci., 39, 791-799, doi:10.1175/15200469(1982)039<0791:TROGWI>2.0.CO;2, 1982.

Holton, J. R. and Alexander, M. J.: The Role of Waves in the Transport Circulation of the Middle Atmosphere, in: Atmospheric Science Across the Stratopause, edited by: Siskind, D. E., Eckermann, S. D., and Summers, M. E., vol. 123 of Geophys. Monogr. Ser., 21-35, AGU, Washington, D.C., USA, doi:10.1029/GM123p0021, 2000.

Keuer, D., Hoffmann, P., Singer, W., and Bremer, J.: Longterm variations of the mesospheric wind field at mid-latitudes, Ann. Geophys., 25, 1779-1790, doi:10.5194/angeo-25-17792007, 2007.

Kudeki, E. and Franke, S. J.: Statistics of momentum flux estimation, J. Atmos. Sol.-Terr. Phy., 60, 1549-1553, doi:10.1016/S1364-6826(98)00104-7, 1998.

Lindzen, R. S.: Turbulence and stress owing to gravity wave and tidal breakdown, J. Geophys. Res., 86, 9707-9714, doi:10.1029/JC086iC10p09707, 1981.

Liu, H.-L., Marsh, D. R., She, C.-Y., Wu, Q., and Xu, J.: Momentum balance and gravity wave forcing in the mesosphere and lower thermosphere, Geophys. Res. Lett., 36, L07805, doi:10.1029/2009GL037252, 2009.

Matthias, V., Hoffmann, P., Manson, A., Meek, C., Stober, G., Brown, P., and Rapp, M.: The impact of planetary waves on the latitudinal displacement of sudden stratospheric warmings, Ann. Geophys., 31, 1397-1415, doi:10.5194/angeo-31-13972013, 2013.
Pancheva, D. V. and Mitchell, N. J.: Planetary waves and variability of the semidiurnal tide in the mesosphere and lower thermosphere over Esrange $\left(68^{\circ} \mathrm{N}, 21^{\circ} \mathrm{E}\right)$ during winter, J. Geophys. Res., 109, A08307, doi:10.1029/2004JA010433, 2004.

Picone, J. M., Hedin, A. E., Drob, D. P., and Aikin, A. C.: NRLMSISE-00 empirical model of the atmosphere: Statistical comparisons and scientific issues, J. Geophys. Res., 107 (A12), 1468, doi:10.1029/2002JA009430, 2002.

Placke, M., Hoffmann, P., Latteck, R., and Rapp, M.: Gravity wave momentum fluxes from MF and meteor radar measurements in the polar MLT region, J. Geophys. Res.-Space, 120, 736-750, doi:10.1002/2014JA020460, 2015.

Reid, I. M. and Vincent, R. A.: Measurements of mesospheric gravity wave momentum fluxes and mean flow accelerations at Adelaide, Australia, J. Atmos. Terr. Phys., 49, 443-460, 1987.

Sato, K.: A statistical study of the structure, saturation and sources of inertio-gravity waves in the lower stratosphere observed with the MU radar, J. Atmos. Terr. Phys., 56, 755-774, doi:10.1016/0021-9169(94)90131-7, 1994.

Singer, W., Latteck, R., and Holdsworth, D. A.: A new narrow beam Doppler radar at $3 \mathrm{MHz}$ for studies of the highlatitude middle atmosphere, Adv. Space Res., 41, 1488-1494, doi:10.1016/j.asr.2007.10.006, 2008.

Vincent, R. A. and Reid, I. M.: HF Doppler Measurements of Mesospheric Gravity Wave Momentum Fluxes, J. Atmos. Sci., 40, 1321-1333, doi:10.1175/15200469(1983)040<1321:HDMOMG>2.0.CO;2, 1983. 